Journal of Mathematics and Statistics 5 (1): 47-53, 2009

ISSN 1549-3644

(C) 2009 Science Publications

\title{
A Numerical Test on the Riemann Hypothesis with Applications
}

\author{
${ }^{1}$ Oladejo, N.K. and ${ }^{2}$ I.A. Adetunde \\ ${ }^{1}$ Department of Applied Mathematics and Computer Science, \\ University for Development Studies Navrongo, Ghana \\ ${ }^{2}$ Department of Mathematics, University of Mines and Technology, Tarkwa, Ghana
}

\begin{abstract}
Problem statement: The Riemann hypothesis involves two products of the zeta function $\zeta(\mathrm{s})$ which are: Prime numbers and the zeros of the zeta function $\zeta(\mathrm{s})$. It states that the zeros of a certain complex-valued function $\zeta(s)$ of a complex number $s \neq 1$ all have a special form, which may be trivial or non trivial. Zeros at the negative even integers (i.e., at $S=-2, S=-4, S=-6 \ldots$ ) are called the non-trivial zeros. The Riemann hypothesis is however concerned with the trivial zeros. Approach: This study tested the hypothesis numerically and established its relationship with prime numbers. Results: Test of the hypotheses was carried out via relative error and test for convergence through ratio integral test was proved to ascertain the results. Conclusion: The result obtained in the above findings and computations supports the fact that the Riemann hypothesis is true, as it assumed a smaller error as possible as $\mathrm{x}$ approaches infinity and that the distribution of primes was closely related to the Riemann hypothesis as was tested numerically and the Riemann hypothesis had a positive relationship with prime numbers.
\end{abstract}

Key words: Riemann hypothesis, zeta function, gamma function, errors, prime, asymptote, integral

\section{INTRODUCTION}

Riemann hypothesis also known as the Riemann zeta hypothesis, first formulated by G.F.B Riemann in 1859, is one of the most famous and important but posed a very difficult problem in mathematics despite attracting concentrated effort from many outstanding mathematicians.

The Riemann hypothesis involves two products of the zeta function $\zeta(\mathrm{s})$ which are: Prime numbers and the zeros of the zeta function $\zeta(\mathrm{s})$.It states that the zeros of a certain complex-valued function $\zeta(\mathrm{s})$ of a complex number $s \neq 1$ all have a special form, which may be trivial or non trivial. Zeros at the negative even integers (i.e., at $\mathrm{S}=-2, \mathrm{~S}=-4, \mathrm{~S}=-6 \ldots$ ) are called the nontrivial zeros. The Riemann hypothesis is however concerned with the trivial zeros and states that:

The real part of any non-trivial zeros of the Riemann zeta function is $1 / 2$ symbolically $\operatorname{Re}(\mathrm{s})=1 / 2$, lying on the so-called critical line. The real part zeros correspond to the zeros of the zeta function on the critical line.

In spite of its simplicity, the Riemann function has turned out to be difficult to Prove. It is however important and of intense interest to number theorist since it is thoroughly entangled with prime numbers.
The study therefore, wish to test Riemann Hypothesis numerically and also to establish the relationship between Riemann Hypothesis and Prime numbers.

The zeta function: The zeta function is an extremely important special function of integration and is intimately related with very deep results surrounding it. The zeta function of one complex variable which is conventionally denoted by $\mathrm{s}$ is defined in various forms including:

- $\zeta(\mathrm{s})=\frac{1}{\Gamma(\mathrm{x})} \int_{0}^{\infty} \frac{\mathrm{U}^{\mathrm{x}-1}}{\mathrm{e}^{\mathrm{u}}-1} \mathrm{du}$

where, $\Gamma(\mathrm{x})$ is the gamma function. By Integrating, we have:

$\zeta(\mathrm{n})=\sum_{\mathrm{k}=1}^{\infty} \frac{1}{\mathrm{k}^{\mathrm{n}}}$

which is sometimes referred to as the P-series.

- $\zeta(\mathrm{z})=\frac{\Gamma(1-\mathrm{Z})}{2 \pi \mathrm{i}} \mathfrak{f} \frac{\mathrm{u}^{\mathrm{z}-1}}{\mathrm{e}^{-\mathrm{u}}-1}$

$\mathrm{du}$ for all $\mathrm{z} \neq 1$

Corresponding Author: N.K. Oladejo, Department of Applied Mathematics and Computer Science, University for Development Studies Navrongo, Ghana 
The gamma function: The gamma function is defined by:

$\Gamma(\mathrm{x})=\mathrm{t}^{\mathrm{x}-1} \mathrm{e}^{-\mathrm{t}} \mathrm{dt}$, for all $\mathrm{X}>0$

Integral: An integral is an anti-derivative. A derivative is the limit of the ratio of the increment of a given function $(y=f(x))$ to the increment of the independent variable ( $x)$ as the latter tends to $\operatorname{zero}(\Delta x \rightarrow 0)$. Note that this hold only when the limit exits. The integral is denoted by $\int f(x) d x$.

Contour integral: This is an integral obtained by contour integration. A particular path in a complex plane used to compute the integral. It can be represented by the notation $\int^{(\alpha)} \mathrm{f}(\mathrm{z}) \mathrm{dz}$ which denote the contour integral of $f(z)$ with contour encircling the point $\alpha$ once in a counterclockwise direction.

Asymptote: Asymptotes have to do with continuous functions with limits involving infinity. It is the behavior of a function such that it increases or decreases without bound, that is neither sided limit exits. Examining $\left(\lim \frac{1}{x}\right.$, for both $x \rightarrow 0^{+}$and $x \rightarrow 0^{-}$, graphically, it is seen that $y=\frac{1}{x}$ approaches the vertical axis $(x=0)$ as $x \rightarrow 0$. This indicates vertical asymptote.

Asymptotes may also be horizontal or slant depending on the axis of approach. Some other forms of asymptotes related to this study are the infinite and infinitesimal asymptotes.

Infinite asymptote: This is useful when analyzing algorithms for efficiency. In a polynomial function e.g. $T(n)=4 n^{2}+2 n+2$ where the value of the function approaches the variable with the highest order. For example in the given function, as $\mathrm{n}$ grows large, the $\mathrm{n}^{2}$ term will come to dominate, so that all other terms can be neglected also the co-efficient turn irrelevant. Then the function $T(n)$ can be said to be asymptotic to $n^{2}$.

Infinitesimal asymptote: In a mathematical function involving an infinite process, e.g., $e^{x}=1+x+\frac{x^{2}}{2 !}+\frac{x^{3}}{3 !} \frac{x^{4}}{4 !} \ldots$, the function could be approximated to a particular term within a particular limit. Thus the above process can be approximated to $e^{x}=1+x+\frac{x^{2}}{2 !} \quad$ as $x \rightarrow 0$. Therefore introducing an amount of error. This means $\mathrm{e}^{\mathrm{x}}$ is asymptotic to $1+x+\frac{x^{2}}{2 !}$.
Error: Error is the accumulated effect of inherent uncertainties in data. Errors are inevitable and unavoidable since most numerical methods give answers that are only by approximation of the true desired value. Simply put error is the difference between the approximated value and the true desired value.

There are various reasons for the introduction of error in a numerical method which identifies the type of error. Error may be due to rounding of numbers with infinite or relatively large number of decimal representations, influence of measurements by temporary disturbance during experiments, by the misuse of applied methods, the representation of an infinite process by a finite process just to mention a few.

Historical background of Reimann hypothesis: The Riemann hypothesis is one of the important open problems of contemporary mathematics. Most Mathematicians believe the Riemann hypothesis to be true. However for over a hundred years, since G.F.B Riemann came out with the hypothesis, many investigators like Leohnard Euler, Artin, Gauss, Chebyshev, Madamard, De la vallee Poussin have made several contributions in the form of proofs and conjectures, but they have not been able to address the problem(s) resulting from the hypothesis ${ }^{[1,2,6]}$. We therefore envisaged that a numerical test could be conducted on the Riemann hypothesis to add some clarity to it and also investigate the relationship between the Riemann hypothesis and the prime numbers, which is the main focus of this study.

\section{MATERIALS AND METHODS}

This study intends to test the Riemann hypothesis by numerical means. It is evident from this research work that many writers for many years have been working on what has now come to be known as the Riemann hypothesis, even before G.F.B Riemann came out with his study propounding the hypothesis in 1859 . These outcomes, which are equivalent to the Riemann hypothesis, are either weaker or stronger as they are either implied by it but does not imply it or vice versa respectively.

Riemann in his study (1859), gave an explicit formula that shows that $\pi(x)=\operatorname{Li}(x)+$ other stuff, even though he could not estimate well enough what the other stuff yielded immediately. Also following the prove of the prime number theorem, by Hadamard and Dela Vallee Poussin, an equivalent form of the Riemann hypothesis was formulated in terms of 
absolute error in the approximation of $\pi(x)$ by $\operatorname{Li}(x)$ as $\pi(\mathrm{x})=\operatorname{Li}(\mathrm{x})+\mathrm{E}(\mathrm{x})$ where $|\mathrm{E}(\mathrm{x})| \leq C \mathrm{C}^{1 / 2} \log \mathrm{x}$ referred to as one of the best possible estimate of the Riemann hypothesis. Hadamard further showed that $|\mathrm{E}(\mathrm{x})| \leq \mathrm{Cxe}^{-\mathrm{c} v \ln \mathrm{x}}$ where $\mathrm{C}$ and $\mathrm{c}$ are positive constants or $\mathrm{E}(\mathrm{x})=\mathrm{O}\left(\mathrm{xe}^{-\mathrm{c} / \ln x}\right)$ when making use of the big $\mathrm{O}$ notation $^{[6,1,3]}$.

Some other equivalents of the Riemann hypothesis have been conjectured and proved. In relation to the prime number theorem, the formulation $\pi(x)-\operatorname{Li}(x)=O\left(x^{1 / 2} \ln x\right)$, is equivalent to the Riemann hypothesis.

In 1908, Lindelof proposed equivalence to the Riemann hypothesis, using classical techniques to prove $^{[3,6]}$. This is refered to as the Lindelof hypothesis which states that $\zeta(1 / 2+i \tau) \mathrm{O}\left(\tau^{\varepsilon)}\right.$ for any $\varepsilon>0$ as $\tau \rightarrow \infty$.

This study indicate that the precise distribution of zeros of $\zeta(\mathrm{s})$ affect the goodness of approximation of $\pi(x)$ by $\operatorname{Li}(x)$. Also if all the zeros of the of $\zeta(\mathrm{s})$ lie on the line $\operatorname{Re}(\mathrm{s})=1 / 2$ as Riemann conjectured, then the error term (the difference $\pi(\mathrm{x})-\operatorname{Li}(\mathrm{x})$ ) is as small as possible. The converse is also known to be true. If the error term has that conjectured smallest possible form, then the Riemann hypothesis is true.

The test of the hypothesis would thus be based mostly on the size of the error term as X increases.

The test of the hypothesis: The asymptotic law of distribution of prime numbers which states that $\lim _{x \rightarrow \infty} \frac{\pi(x)}{x / \ln x}=1$, restated as $\pi(x) \sim \frac{x}{\ln x}$, further states that $\frac{x}{\ln x}$ approximates $\pi(x)$ in the sense that the relative error of the approximation approaches 0 as $\mathrm{x} \rightarrow \infty$. This was proved by Chebychev that $\pi(\mathrm{x}) \sim \operatorname{Li}(\mathrm{x})$ since $\operatorname{Li}(x) \sim \frac{x}{\ln x}$. Recall that $\pi(x) \sim \operatorname{Li}(x)$ is a consequence of $\psi(\mathrm{x}) \sim \mathrm{x}$. This is as a result of $\psi(\mathrm{x})$ which grows just a little faster than $\pi(\mathrm{x})$, thus $\operatorname{Li}(\mathrm{x}) \sim \frac{\mathrm{x}}{\ln \mathrm{x}}$ implying $\pi(\mathrm{x}) \sim \frac{\mathrm{x}}{\ln \mathrm{x}}$.

The first test would therefore be based on $\pi(\mathrm{x}) \sim \frac{\mathrm{x}}{\ln \mathrm{x}}$, in relation to the fact that the error term is as small as possible and that the error approaches zero as $\mathrm{x}$ approaches infinity.

$$
\begin{array}{lll}
\text { For } x & =10, \pi(x) & =4 \\
\frac{x}{\ln x} & =\frac{10}{\ln 10} & =4.342944819 \\
\text { Relative error } & =\frac{|4-4.342944819|}{4} & =0.08573620476
\end{array}
$$

$$
\begin{aligned}
& \text { For } \mathrm{x} \quad=100, \pi(\mathrm{x}) \quad=25 \\
& \frac{\mathrm{x}}{\ln \mathrm{x}} \quad=\frac{100}{\ln 100} \quad=21.714724 \\
& \text { Relative error }=\frac{|25-21.714724|}{25}=0.131411036 \\
& \text { For } \mathrm{x} \quad=1000, \pi(\mathrm{x}) \quad=168 \\
& \frac{\mathrm{x}}{\ln \mathrm{x}} \quad=\frac{1000}{\ln 1000} \quad=144.7648273 \\
& \text { Relative error }=\frac{|168-144.76482731|}{168}=0.138
\end{aligned}
$$

The Table 1 shows the relative error using the asymptotic law of distribution.

The goodness of the approximation of $\pi(\mathrm{x})$ by $\operatorname{Li}(\mathrm{x})$ is said to be affected by the distribution of the zeros of $\zeta(\mathrm{s})$, that is if all zeros of $\zeta(\mathrm{s})$, lie on the line $\operatorname{Re}(s)=1 / 2$ as conjectured by Riemann, then the error term must be as small as possible. The main conjecture, equivalent to the Riemann hypothesis, is in terms of the absolute error in the approximation of $\pi(\mathrm{x})$ by $\operatorname{Li}(\mathrm{x})$ as shown in $\pi(x)=\operatorname{Li}(x)+E(x)$ where $E(x)$ is the absolute error and can be represented in the following forms:

$|E(x)| \leq C e^{-c \sqrt{\operatorname{lin} x}}$ or $\frac{E(x)}{x} \leq \mathrm{Ce}^{-c \sqrt{\ln x}}$

In terms of relative error:

$|\mathrm{E}(\mathrm{x})|=\mathrm{O}\left(\mathrm{xe}^{-\mathrm{c} \sqrt{\ln x}}\right)$ or $\frac{\mathrm{E}(\mathrm{x})}{\mathrm{x}}=\mathrm{O}\left(\mathrm{e}^{-\mathrm{c} \sqrt{\ln x}}\right)$

Table 1: The Relative error using the asymptotic law of distribution

\begin{tabular}{llll}
\hline $\mathrm{X}$ & $\pi(\mathrm{x})$ & $\mathrm{x} / \ln \mathrm{x}$ & Relative Error \\
\hline $10^{4}$ & 1229 & 1085.736205 & 0.116566940210 \\
$10^{5}$ & 9592 & 8685.889638 & 0.094465217050 \\
$10^{6}$ & 78498 & 72382.41365 & 0.077907543500 \\
$10^{7}$ & 664579 & 620420.6884 & 0.066455416100 \\
$10^{8}$ & 5761455 & 5428681.024 & 0.057758866968 \\
$10^{9}$ & 50847534 & 4825494.43 & 0.050987557550 \\
$10^{10}$ & 455052511 & 434294481.9 & 0.045616777390 \\
$10^{11}$ & 4118054813 & 3948131654 & 0.041262966790 \\
$10^{12}$ & 37607912018 & $3.61912 * 10^{10}$ & 0.037670402430 \\
$10^{13}$ & 346065536839 & $3.34072 * 10^{11}$ & 0.034654876390 \\
$10^{14}$ & 3204941750802 & $3.10210^{*} 10^{12}$ & 0.032087487500 \\
$10^{15}$ & 29844570422669 & $2.89529 * 10^{13}$ & 0.029874947020 \\
$10^{16}$ & 279238341033925 & $2.71434 * 10^{14}$ & 0.027948489350 \\
$10^{17}$ & 2623557157654233 & $2.55467 * 10^{15}$ & 0.026255854120 \\
$10^{18}$ & 24739954287740860 & $2.41274 * 10^{16}$ & 0.024756838800 \\
$10^{19}$ & 234057667276344607 & $2.28576^{*} 10^{17}$ & 0.023419972380 \\
$10^{20}$ & 2220819602560918840 & $2.17147 * 10^{18}$ & 0.022202613600 \\
$10^{21}$ & 211227269486018731928 & $2.06806 * 10^{19}$ & 0.021137604670 \\
$10^{22}$ & 201467286689315906290 & $1.97440 * 10^{20}$ & 0.020155648930 \\
$10^{23}$ & 1925320391606818006727 & $1.88823 * 10^{21}$ & 0.019260956860 \\
\hline
\end{tabular}


The above is enough to test the Riemann hypothesis since it implies that the relative error $\frac{\mathrm{E}(\mathrm{x})}{\mathrm{x}} \rightarrow 0$ as $\mathrm{x} \rightarrow \infty$ :

- $\Rightarrow$ Relative error $\leq 5 \mathrm{e}^{-3 \sqrt{\ln 10}}=0.05271561984$ For $\mathrm{X}=10^{2}$

- Relative error $\leq 5 \mathrm{e}^{-3 \sqrt{\ln 10^{2}}} \quad=0.007998828653$ For $\mathrm{X}=10^{3}$

- Relative error $\leq 5 \mathrm{e}^{-3 \sqrt{\ln 1000}} \quad=0.001882142088$

The Table 2 shows the computation of Relative error using Eq. 1:

Hence applying Eq. 2, i.e.:

$$
\frac{E(x)}{x}=O\left(e^{-c \sqrt{\ln x}}\right)
$$

For $\mathrm{X}=10$ :

Relative error $=\mathrm{O}\left(\mathrm{e}^{-3 \sqrt{\ln 10}}=0.01054312397\right.$

For $\mathrm{X}=100$ :

Relative error $=\mathrm{O}\left(\mathrm{e}^{-3 \sqrt{\ln 100}}\right)=0.001599765731$

The Table 3 shows the computation of relative error using Eq. 2.

The Riemann hypothesis ${ }^{[4]}$ is so important to number theorist and implies the smallest possible error estimate in the prime number theorem.

Table 2: The computation of relative error using Eq. 1 above, that is $\left.\mathrm{Ce}^{\sqrt{\ln \mathrm{x}}}\right)$

\begin{tabular}{lll}
\hline $\mathrm{X}$ & $\mathrm{C} \sqrt{\ln \mathrm{x}}$ & Relative error $\left(\mathrm{Ce}^{\sqrt{\ln \mathrm{x}}}\right)$ \\
\hline $10^{4}$ & $3.034854259 \mathrm{c}$ & $5.5578 \times 10^{-4}$ \\
$10^{5}$ & $3.393070212 \mathrm{c}$ & $1.8975 \times 10^{-4}$ \\
$10^{6}$ & $3.716922189 \mathrm{c}$ & $7.1821 \times 10^{-5}$ \\
$10^{7}$ & $4.014734817 \mathrm{c}$ & $2.9392 \times 10^{-5}$ \\
$10^{8}$ & $4.291932053 \mathrm{c}$ & $1.2796 \times 10^{-5}$ \\
$10^{9}$ & $4.552281388 \mathrm{c}$ & $5.8597 \times 10^{-6}$ \\
$10^{10}$ & $4.798525912 \mathrm{c}$ & $2.7993 \times 10^{-6}$ \\
$10^{11}$ & $5.032736435 \mathrm{c}$ & $1.3864 \times 10^{-6}$ \\
$10^{12}$ & $5.25652177 \mathrm{c}$ & $7.0841 \times 10^{-7}$ \\
$10^{13}$ & $5.471161322 \mathrm{c}$ & $3.7212 \times 10^{-7}$ \\
$10^{14}$ & $5.677692428 \mathrm{c}$ & $2.0026 \times 10^{-7}$ \\
$10^{15}$ & $5.876970001 \mathrm{c}$ & $1.1014 \times 10^{-7}$ \\
$10^{16}$ & $6.069708158 \mathrm{c}$ & $6.1779 \times 10^{-8}$ \\
$10^{17}$ & $6.256512334 \mathrm{c}$ & $2.5274 \times 10^{-8}$ \\
$10^{18}$ & $6.437898079 \mathrm{c}$ & $2.0471 \times 10^{-8}$ \\
$10^{19}$ & $6.614311511 \mathrm{c}$ & $1.2058 \times 10^{-8}$ \\
$10^{20}$ & $6.786140424 \mathrm{c}$ & $7.2014 \times 10^{-9}$ \\
$10^{21}$ & $6.953724682 \mathrm{c}$ & $4.3558 \times 10^{-9}$ \\
$10^{22}$ & $7.117364133 \mathrm{c}$ & $2.6660 \times 10^{-9}$ \\
$10^{23}$ & $7.277324861 \mathrm{c}$ & $1.6499 \times 10^{-9}$ \\
\hline
\end{tabular}

It gives as much information as possible about the distribution of prime numbers and the zeros of the zeta function. $|\pi(\mathrm{x})-\mathrm{Li}(\mathrm{x})|=\mathrm{O}\left(\mathrm{x}^{1 / 2} \ln \mathrm{x}\right)$ as $\mathrm{x} \rightarrow \infty$ has been proven to be equivalent to the Riemann hypothesis. Knowledge of the distribution of primes is required for the derivative of the best possible estimates.

The Table 4 shows the error.

Besides, Riemann ${ }^{[1,4,5]}$ went on to give an explicit formula for $\pi(\mathrm{x})$, the number of primes not greater than $\mathrm{X}$. Reviewing what have been already said about $\pi(\mathrm{x})$,

Table 3: The computation of relative error using Eq. 2 above, that is $\mathrm{O} \mathrm{e}^{-\mathrm{c} \sqrt{\ln \mathrm{x}}}$

\begin{tabular}{ll}
\hline $\mathrm{X}$ & Relative error $\left\{\mathrm{O} \mathrm{e}^{-\mathrm{c} \sqrt{\ln \mathrm{x}}}\right)$ \\
\hline $10^{3}$ & 0.0003764284176000000 \\
$10^{4}$ & 0.0001111574630000000 \\
$10^{5}$ & 0.0000379511543600000 \\
$10^{6}$ & 0.0000143642717000000 \\
$10^{7}$ & 0.0000058785263330000 \\
$10^{8}$ & 0.0000025592503930000 \\
$10^{9}$ & 0.0000011719469120000 \\
$10^{10}$ & 0.0000005598607546000 \\
$10^{11}$ & 0.0000002772879598000 \\
$10^{12}$ & 0.0000001416983536000 \\
$10^{13}$ & 0.0000000744242531300 \\
$10^{14}$ & 0.0000000400523983000 \\
$10^{15}$ & 0.0000000220289133400 \\
$10^{16}$ & 0.0000000123559815800 \\
$10^{17}$ & 0.0000000070549453290 \\
$10^{18}$ & 0.0000000040942010740 \\
$10^{19}$ & 0.0000000024116985780 \\
$10^{20}$ & 0.0000000014402901170 \\
$10^{21}$ & 0.0000000008711786544 \\
$10^{22}$ & 0.0000000005332171488 \\
$10^{23}$ & 0.0000000003299847798 \\
\hline
\end{tabular}

Table 4: The error computed as $\mathrm{x} \rightarrow \infty$ when we have $\mathrm{X}^{1 / 2} \ln \mathrm{X}$

\begin{tabular}{llrl}
\hline$X$ & $X^{1 / 2}$ & LnX & Error $\left(X^{1 / 2} \ln X\right)$ \\
\hline 10 & 3.16227766 & 2.3025850930 & 7.2814134 \\
$10^{2}$ & 10 & 4.6051701860 & 46.05170186 \\
$10^{3}$ & 31.6227766 & 6.9077552279 & 218.442402 \\
$10^{4}$ & 100 & 9.2103403720 & 921.0340372 \\
$10^{5}$ & 316.227766 & 11.5129254600 & 3640.7067 \\
$10^{6}$ & 1000 & 13.8155105600 & 13815.51056 \\
$10^{7}$ & 3162.27766 & 16.1180956500 & 50969.8938 \\
$10^{8}$ & 10000 & 18.4206807400 & 184206.8074 \\
$10^{9}$ & 31622.7766 & 20.7232658400 & 655327.206 \\
$10^{10}$ & 100000 & 23.0258509300 & 2302585.093 \\
$10^{11}$ & 316227.766 & 25.3284360200 & 8009554.74 \\
$10^{12}$ & 1000000 & 27.6310211200 & 27631021.12 \\
$10^{13}$ & 3162277.66 & 29.9336062100 & 94658374.2 \\
$10^{14}$ & 10000000 & 32.2361913000 & 322361913 \\
$10^{15}$ & 31622776.6 & 34.5387763900 & 1092212010 \\
$10^{16}$ & 100000000 & 36.8413614900 & 3684136149 \\
$10^{17}$ & 316227766 & 39.1439465800 & $1.237840278 \times 10^{10}$ \\
$10^{18}$ & 1000000000 & 41.4465316700 & $4.144653167 \times 10^{10}$ \\
$10^{19}$ & 3162277660 & 43.7491167700 & $1.383468546 \times 10^{11}$ \\
$10^{20}$ & 10000000000 & 46.0517018600 & $4.605170186 \times 10^{11}$ \\
$10^{21}$ & 31622776600 & 48.3542869500 & $1.529096814 \times 10^{12}$ \\
$10^{22}$ & 316227766000 & 52.9594571400 & $5.065687205 \times 10^{12}$ \\
\hline
\end{tabular}


based on the probabilistic ideas as well as extensive calculations, Gauss conjectured ${ }^{[5]}$ that $\pi(x) \sim \frac{x}{\ln x}$. A similar function which gives a slightly better result, both theoretically and computationally is the logarithmic integral:

$$
\operatorname{Li}(x)=\int_{0}^{x}(\ln t)^{-1} d t .
$$

Nelson showed and Ramanujan ${ }^{[4]}$ independently discovered that:

$\operatorname{Li}(x)=\int_{0}^{x}(\ln t)^{-1} d t=\ln \ln x+\ln x+\frac{(\ln x)^{2}}{2 \times 2 !}+\frac{(\ln x)^{3}}{3 \times 3 !}+\ldots \ldots \ldots \ldots$

which can be expressed as $\int(\ln x)^{-1}=\ln \ln x+\sum_{n=1}^{\infty} \frac{(\ln x)^{n}}{n \times n !}$.

It is observed that the expression for $\operatorname{Li}(\mathrm{x})$ involves a geometric progression (series). The sum could therefore be computed depending on: whether the series diverges or converges and whether the common ratio of the geometric progression is less than 1 or not.

There is therefore the need to test for convergence of the series and to find out whether the common ratio is less than 1 .

Test for convergence of $\sum_{n=1}^{\infty} \frac{(\ln x)^{n}}{n \times n !}$ ratio test:

$$
\begin{aligned}
& a_{n}=\frac{(\ln x)^{n}}{n \times n !}, \\
& a_{n+1}=\frac{(\ln x)^{n+1}}{(n+1)(n+1) !} \\
&= \frac{(\ln x)^{n} \bullet \ln x}{n n !+n+n !+1} \\
& \lim _{n \rightarrow \infty}\left|\frac{a_{n+1}}{a_{n}}\right|=\lim _{n \rightarrow \infty}\left|\frac{(\ln x)^{n} \bullet \ln x}{n n !+n !+n+1} / \frac{(\ln x)^{n}}{n \times n !}\right| \\
&=\lim _{n \rightarrow \infty}\left|\frac{(\ln x)^{n} \ln x}{n n !+n !+n+1} \times \frac{n n !}{(\ln x)^{n}}\right| \\
&=\lim _{n \rightarrow \infty} \mid \frac{(\ln x)}{n n !\left(1+\frac{1}{n}+\frac{1}{n !}+\frac{1}{n n !}\right)} \times n n ! \\
&=\lim _{n \rightarrow \infty}\left|\frac{\ln x}{1+\frac{1}{n}+\frac{1}{n !}+\frac{1}{n n !} \mid=\ln x}\right|
\end{aligned}
$$

Therefore the radius of convergence is $\ln (-x, x)$.

Even though the limit of the geometric series exists and there is a radius of convergence, the common ratio, $\frac{(n-1) \ln x}{n^{2}}$, is greater than one for all $x>2$ and $n>1$. The geometric progression is thus said to be divergent. It is however observed that, as $n$ increases, $\frac{(\ln x)^{n}}{n \times n !}$ increases to a maximum and then begins to decrease indefinitely, the cause for which the series diverges. However, at a particular $n$ value the value for $\frac{(\ln x)^{n}}{n n !}$ falls below 1 . For the purpose of this study, these values would be considered insignificant, likewise the sum of $\frac{(\ln x)^{n}}{n n !}$, for all $n$ greater than this particular $n$ (i.e., $\sum_{n=m}^{\infty} \frac{(\ln x)^{n}}{n n !}$, where $m$ is the $n$ for which $\left.\frac{(\ln x)^{n}}{n n !}<1\right)$. For $x$ values of $10,10^{2}, 10^{3}, 10^{4}, \ldots, 10^{13}$, it is observed that $\mathrm{n} \leq 2,7$, $13,18,24,30,36,42,49,55,61,67,73$ respectively seem to have values greater than 1 , with an average difference of 6 . Sum up to these n's (i.e., m-1) would therefore be considered the sum of the various X's listed above respectively. The series is therefore considered to be convergent based on these conditions.

By the use of asymptotes, it can be said that the sum of a particular value of $\mathrm{X}$ is infinitesimally asymptotic to the partial sum up to a particular $n=m-1$.

E.g., For $\mathrm{x}=10^{3}$ :

$$
\begin{aligned}
\mathrm{Sn} & =\sum_{\mathrm{n}=1}^{13} \frac{(\ln \mathrm{x})^{\mathrm{n}}}{\mathrm{nn} !}+\mathrm{O}\left[(\ln \mathrm{x})^{14}\right] \\
& =\operatorname{Inx}+\frac{(\ln \mathrm{x})^{2}}{2 \times 2 !}+\frac{(\ln \mathrm{x})^{3}}{3 \times 3 !}+\ldots \ldots \ldots+\frac{(\ln \mathrm{x})^{13}}{13 \times 13 !}+\mathrm{O}\left[(\ln \mathrm{x})^{14}\right]
\end{aligned}
$$

For $\mathrm{X}=10^{6}$ :

$$
\begin{aligned}
\mathrm{Sn} & =\sum_{\mathrm{n}=1}^{30} \frac{(\ln \mathrm{x})^{\mathrm{n}}}{\mathrm{nn} !}+\mathrm{O}\left[(\ln \mathrm{x})^{\mathrm{n}+1}\right] \\
& =\ln \mathrm{x}+\frac{(\ln \mathrm{x})^{2}}{2 \times 2 !}+\frac{(\ln \mathrm{x})^{3}}{3 \times 3 !}+\ldots \ldots \ldots+\frac{(\ln \mathrm{x})^{30}}{30 \times 30 !}+\mathrm{O}\left[(\ln \mathrm{x})^{31}\right]
\end{aligned}
$$

For $\mathrm{X}=10^{9}$ :

$$
\begin{aligned}
\mathrm{Sn} & =\sum_{\mathrm{n}=1}^{49} \frac{(\ln X)^{\mathrm{n}}}{\mathrm{n} \times \mathrm{n} !}+\mathrm{O}\left[(\ln \mathrm{x})^{\mathrm{n}+1}\right] \\
& =\ln \mathrm{x}+\frac{(\ln \mathrm{x})^{2}}{2 \times 2 !}+\frac{(\ln \mathrm{x})^{3}}{3 \times 3 !}+\ldots \ldots \ldots \ldots+\frac{(\ln \mathrm{x})^{49}}{49 \times 49 !}+\mathrm{O}\left[(\ln \mathrm{x})^{50}\right]
\end{aligned}
$$


J. Math. \& Stat., 5 (1): 47-53, 2009

Table 5: The computations of the Relative error using the logarithmic integral

\begin{tabular}{lllrl}
\hline $\mathrm{X}$ & $\pi(\mathrm{x})$ & $\mathrm{m}-1$ & $\mathrm{Li}(\mathrm{x})=\int \frac{\mathrm{dt}}{\ln \mathrm{t}}=\ln \ln \mathrm{x}+\sum_{\mathrm{n}=1}^{\mathrm{m}-1} \frac{(\ln \mathrm{x})^{\mathrm{n}}}{\mathrm{nn} !}$ & Relative error \\
\hline $10^{4}$ & 1229 & 18 & 1243.9850000000000 & 0.01219000000 \\
$10^{5}$ & 9592 & 24 & 9627.7390000000000 & 0.00370000000 \\
$10^{6}$ & 78498 & 30 & 78625.2700000000000 & 0.00162000000 \\
$10^{7}$ & 664579 & 42 & 664916.278500000000 & 0.00050700000 \\
$10^{8}$ & 5761455 & 49 & 5762207.1460000000000 & 0.00013050000 \\
$10^{9}$ & 50847534 & 55 & 450849233.7600000000000 & 0.00003342000 \\
$10^{10}$ & 445052511 & 61 & 4118066398.10000000000000 & 0.00000681700 \\
$10^{11}$ & 4118054813 & 67 & 37607950279.2195000000000 & 0.00000281320 \\
$10^{12}$ & 37607912018 & 73 & 346065645808.3200000000000 & 0.00000101730 \\
$10^{13}$ & 346065536839 & & & 0.00000031496 \\
\hline
\end{tabular}

Table 6: The summary of errors computed from the results

\begin{tabular}{|c|c|c|c|c|c|}
\hline $\mathrm{X}$ & $\pi(x)-\frac{x}{\ln x}$ & $\mathrm{O}\left(\mathrm{e}^{-\mathrm{c} \sqrt{\ln x}}\right)$ & $\mathrm{Ce}^{-c \sqrt{\ln x}}$ & $\pi(\mathrm{x})-\int \frac{\mathrm{dt}}{\ln \mathrm{t}}$ & $x^{1 / 2} \operatorname{In} x$ \\
\hline 10 & $8.57 \times 10^{-2}$ & $1.05 \times 10^{-2}$ & $5.27 \times 10^{-2}$ & 0.115 & 7.281 \\
\hline $10^{2}$ & $1.31 \times 10^{-1}$ & $1.59 \times 10^{-3}$ & $7.99 \times 10^{-3}$ & $1.379 \times 10^{-1}$ & 46.052 \\
\hline $10^{3}$ & $1.38 \times 10^{-1}$ & $3.76 \times 10^{-4}$ & $1.88 \times 10^{-3}$ & $4.907 \times 10^{-2}$ & 218.442 \\
\hline $10^{4}$ & $1.11 \times 10^{-1}$ & $1.11 \times 10^{-4}$ & $5.56 \times 10^{-4}$ & $1.2 \times 10^{-2}$ & 921.034 \\
\hline $10^{5}$ & $9.44 \times 10^{-2}$ & $3.79 \times 10^{-5}$ & $1.89 \times 10^{-4}$ & $3.7 \times 10^{-3}$ & 3640.707 \\
\hline $10^{6}$ & $7.79 \times 10^{-2}$ & $1.43 \times 10^{-5}$ & $7.18 \times 10^{-5}$ & $1.62 \times 10^{-3}$ & 13815.511 \\
\hline $10^{7}$ & $6.64 \times 10^{-2}$ & $5.87 \times 10^{-6}$ & $2.93 \times 10^{-5}$ & $5.07 \times 10^{-4}$ & 50969.893 \\
\hline $10^{8}$ & $5.77 \times 10^{-2}$ & $2.55 \times 10^{-6}$ & $1.27 \times 10^{-5}$ & $1.305 \times 10^{-4}$ & 184206.807 \\
\hline $10^{9}$ & $5.09 \times 10^{-2}$ & $1.17 \times 10^{-6}$ & $5.86 \times 10^{-6}$ & $3.34 \times 10^{-5}$ & 655327.206 \\
\hline $10^{10}$ & $4.56 \times 10^{-2}$ & $5.59 \times 10^{-7}$ & $2.79 \times 10^{-6}$ & $6.817 \times 10^{-6}$ & 2302585.093 \\
\hline $10^{11}$ & $4.12 \times 10^{-2}$ & $2.77 \times 10^{-7}$ & $1.38 \times 10^{-6}$ & $2.813 \times 10^{-6}$ & 8009554.740 \\
\hline $10^{12}$ & $3.76 \times 10^{-2}$ & $1.41 \times 10^{-7}$ & $7.08 \times 10^{-7}$ & $1.017 \times 10^{-6}$ & 27631021.120 \\
\hline $10^{13}$ & $3.46 \times 10^{-2}$ & $7.44 \times 10^{-8}$ & $3.72 \times 10^{-7}$ & $3.149 \times 10^{-7}$ & 94658374.200 \\
\hline
\end{tabular}

It thus implies that $\operatorname{Li}(\mathrm{x})=\ln \ln \mathrm{x}+\left\{\sum_{\mathrm{n}=1}^{\mathrm{m}-1} \frac{(\ln \mathrm{x})^{\mathrm{n}}}{\mathrm{nn} !}\right.$ $\left.+\mathrm{O}\left[(\ln \mathrm{x})^{\mathrm{m}}\right]\right\}$ for a particular $\mathrm{X}$, where $\mathrm{O}\left[(\ln \mathrm{x})^{\mathrm{m}}\right]$ are the insignificant sums.

$$
\text { E.g., } \begin{aligned}
\text { Li (10) } & =\ln \ln 10+\sum_{n=1}^{2} \frac{(\ln 100)^{n}}{n \times n !} \\
& =0.8340324452+3.6279 \\
& =4.4619
\end{aligned}
$$

Absolute error $=|4-4.4619|=0.4619$
Relative error $=\frac{0.4619}{4}=0.115$

$\operatorname{Li}(100)=\ln \ln \mathrm{x}+\sum_{\mathrm{n}=1}^{7} \frac{(\ln 100)^{\mathrm{n}}}{\mathrm{n} \times \mathrm{n} !}$

$=1.527279626+26.9227$

$=28.4499$

Absolute error $=|25-28.4499|=3.4499$

Relative error $=\frac{3.4499}{25}=0.13799$ $\begin{aligned} \operatorname{Li}(1000) & =\ln \ln x+\sum_{n=1}^{13} \frac{(\ln 1000)^{\mathrm{n}}}{\mathrm{nn} !} \\ & =176.244\end{aligned}$

Absolute error $=|168-176.244|=8.244$

Relative error $=\frac{8.244}{168} \quad=0.04907$

The Table 5 shows the computations of the Relative error using the logarithmic integral.

\section{RESULTS AND DISCUSSION}

Numerical investigations are presented in this section to illustrate the aforementioned different methods used respectively i.e., (i) $\pi(\mathrm{x})-\frac{\mathrm{x}}{\ln \mathrm{x}}$, (ii) $\mathrm{O}\left(\mathrm{e}^{-\mathrm{c} \sqrt{\ln \mathrm{x}}}\right)$, (iii) $\mathrm{Ce}^{-\mathrm{c} \sqrt{\ln x}}$, (iv) $\pi(\mathrm{x})-\int \frac{\mathrm{dt}}{\ln \mathrm{t}}$ and (v) $\mathrm{x}^{1 / 2} \ln \mathrm{x}$. Evidently from Table 6, considering the various methods, it was observed that for $x^{1 / 2} \ln x$ (i.e., the fifth method (v)) the error decreasing as $x$ grow larger, assuming a smaller and smaller magnitude as possible.

The Table 6 shows the summary of errors computed from the results. 
Table 7: The hypothesized relation with the difference of six (6)

\begin{tabular}{lllll}
\hline $\mathrm{X}$ & $\pi(\mathrm{x})$ & $\mathrm{m}-1$ & $\mathrm{Li}(\mathrm{x})$ & Error \\
\hline 5 & 3 & 1 & 2.0853 & 0.3049 \\
11 & 5 & 2 & 4.7099 & 0.0580 \\
17 & 7 & 3 & 7.1447 & 0.0206 \\
23 & 9 & 4 & 9.4552 & 0.0505 \\
29 & 10 & 4 & 11.5979 & 0.1597 \\
35 & 11 & 4 & 13.0918 & 0.1901 \\
41 & 13 & 5 & 14.4764 & 0.1136 \\
46 & 14 & 5 & 15.5630 & 0.1116 \\
52 & 15 & 5 & 16.7996 & 0.1199 \\
58 & 16 & 6 & 19.0112 & 0.1882 \\
63 & 18 & 6 & 20.0818 & 0.1156 \\
69 & 19 & 6 & 21.3260 & 0.1224 \\
72 & 20 & 6 & 21.7726 & 0.0806 \\
78 & 21 & 6 & 23.1196 & 0.1009 \\
84 & 32 & 6 & 24.2725 & 0.0553 \\
90 & 24 & 7 & 26.4538 & 0.1022 \\
96 & 24 & 7 & 27.6594 & 0.1524 \\
102 & 26 & 7 & 28.8417 & 0.1092 \\
108 & 28 & 7 & 30.0023 & 0.0715 \\
114 & 30 & 7 & 31.1428 & 0.0380 \\
120 & 30 & 7 & 32.2645 & 0.0754 \\
126 & 30 & 7 & 33.3686 & 0.1122 \\
132 & 32 & 8 & 35.4579 & 0.1080 \\
138 & 33 & 8 & 36.6052 & 0.1092 \\
144 & 34 & 8 & 37.7390 & 0.1099 \\
150 & 35 & 8 & 38.8603 & 0.1102 \\
156 & 36 & 8 & 39.9694 & 0.1102 \\
162 & 37 & 8 & 41.0671 & 0.1099 \\
168 & 39 & 8 & 42.1538 & 0.0808 \\
174 & 40 & 8 & 43.2300 & 0.0807 \\
180 & 41 & 8 & 44.2963 & 0.0803 \\
186 & 42 & 8 & 45.3528 & 0.0798 \\
192 & 43 & 8 & 46.4002 & 0.0790 \\
198 & 45 & 8 & 47.4387 & 0.0541 \\
\hline & & & &
\end{tabular}

Application of the hypothesized relation: The relation $\operatorname{Li}(\mathrm{x})=\int_{0}^{\mathrm{x}} \frac{\mathrm{dt}}{\ln \mathrm{t}}=\ln \ln \mathrm{x}=\sum_{\mathrm{n}=1}^{\mathrm{m}-1} \frac{(\ln \mathrm{x})^{\mathrm{n}}}{\mathrm{nn} !}+\mathrm{O}\left[(\ln \mathrm{x})^{\mathrm{m}}\right]$ has been proven to be useful in estimating the values of the summations for the multiples of 10 . It would be more useful if it is able to estimate sums for values of $x$, which are not multiples of 10 . It is therefore necessary to test to find out if the relation would be useful in this regard. The test would be limited to $5 \leq \mathrm{x} \leq 200$ with a difference of 6.

The Table 7 shows the hypothesized relation with the difference of six.

\section{CONCLUSION}

The various errors have been summarized in Table 6. It is evident from the Table 6 that the various errors considered except for $\mathrm{x}^{\frac{1}{2}} \ln \mathrm{x}$ is observed to be decreasing as $\mathrm{X}$ grows larger (i.e., approaching infinity), assuming a smaller and smaller magnitude as possible. This is similar to what have been proved earlier on, on this study work, that the Riemann hypothesis is true if the error term is as small as possible (or vice versa). For that matter, the zeros of the zeta function are as close as possible to the so-called critical line $\operatorname{Re}(\mathrm{s})=1 / 2$.

Besides, Table 7 indicates the errors computed using the relation $\operatorname{Li}(\mathrm{x})=\int_{0}^{\mathrm{x}} \frac{\mathrm{dt}}{\ln \mathrm{t}}=\ln \ln \mathrm{x}+\sum_{\mathrm{n}=1}^{\mathrm{m}-1} \frac{(\ln \mathrm{x})^{\mathrm{n}}}{\mathrm{nn} !}$, for values of $\mathrm{x}$ which are not multiples of ten. It is evident from Table 7 that the error does not follow any particular pattern (increasing or decreasing but both). The sum of the series for $\mathrm{x}$ up to $\mathrm{m}-1$ is monotonic as $\mathrm{x}$ approaches infinity. However, for $\pi(\mathrm{x})$ (the number of primes less than or equal to $\mathrm{x}$ ) does not necessarily increase for every $\mathrm{x}$, likewise $\mathrm{m}^{-1}$ (the maximum value for $n$ for which $\frac{(\ln x)^{n}}{n n !}$ is greater than or equal to 1 Since relative error is the ratio of the difference between the number of primes $\pi(x)$ and the sum $\operatorname{Li}(\mathrm{x})(|\pi(\mathrm{x})-\operatorname{Li}(\mathrm{x})|$ and the number of primes, the number of primes not increasing whiles $\operatorname{Li}(\mathrm{x})$ increases, thus affects the error. Therefore the error which is the bases for the authentication of the Riemann hypothesis is determined by the distribution of prime number.

The result obtained in the above findings and computations supports the fact that the Riemann hypothesis is true, as it assumes a smaller error as possible as $\mathrm{x}$ approaches infinity and that the distribution of primes is closely related to the Riemann hypothesis as was tested numerically and the Riemann hypothesis has a positive relationship with prime numbers.

\section{REFERENCES}

1. Alex Himmonas and Alan Howard, 2003. Calculus, Ideals and Application. Lauurina Rosatone. Paper Back Publisher, Wiley, New York, USA., ISBN: 978-0471-40145-2, pp: 832.

2. Borwein, J. and D. Bailey, 2003. Mathematics by Experiment: Plausible Reasoning in the 21st Century AK. Peters, ISBN: 1-56881-211-6, pp: 288.

3. Chaitins, G.J., 2003. From Philosophy to Programm size. Tallin, 8th Estonian Winter School in Computer Science, ESWSCS '03. Institute of Cybernetics, ISBN: 9985894553, pp: 41-60.

4. Rockmore, D., 2005. Stalking the Riemmann Hypothesis. The Quest to Find Hidden Law of Prime Numbers. Vintage Books, ISBN: 10: 037542136X, pp: 304.

5. Titchmansh, E.C., 1986. Theory of the Riemann Zeta Function. 2nd Revised Edn., Oxford University Press, ISBN: 0198533691, pp: 412.

6. Zeidler, E., W. Hackburch and H.R. Schwarz, 2003. Oxford Guide to Mathematics. Oxford University Press, pp: 112-135. 